\title{
El sujeto de investigación: Aproximaciones a su estudio
}

\author{
Fernando Lolas Stepke
}

Tras definir la investigación biomédica y psicosocial como una práctica social que involucra a numerosos actores, este artículo centra su atención en los sujetos (o participantes) en experimentos, observaciones, estudios y ensayos farmacológicos, destacando la escasa atención que han recibido sus atributos, a pesar del reconocimiento explícito e implícito de la importancia que estos tienen para la generación de conocimiento. Se destaca la necesidad de precisar los rasgos y caracteres del "voluntario" y su influencia decisiva en estudios psicológicos y sociales.

Investigación / sujetos experimentales / voluntarios / bioética

\section{The person as a research subject: approaches to his study}

After conceptualizing biomedical and psychosocial research as a social practice involving numerous actors, this article concentrates on subjects (or participants) in experiments, observations, studies and pharmacological trials, emphasizing the scarce attention which their attribvutes have received despite the implicit and explicit recognition of their importance in knowledge production. The need to specify traits and characters of the "volunteer" and their decisive influence on psychological and social studies is emphasized.

Research / experimental subjects / volunteers / bioethics

Correo electrónico: flolas@uchile.cl 
LA INVESTIGACIÓN TECNOCIENTÍFICA COMO PRÁCTICA SOCIAL

La bioética contemporánea nació de una legítima preocupación por el bienestar de las personas en el contexto de la investigación biomédica y psicosocial. Los casos emblemáticos aludidos en las palabras Nürenberg, Tuskegee, Helsinki, Belmont, entre muchas otras, reflejan la tensión existente entre el bienestar de las personas, el progreso científico y el bien común (Lolas, 2001a). Entre la philo-tekhnía, el amor al arte, la philo-anthropía, el amor a la humanidad, la autho-philia, el amor a sí mismos de los investigadores, han existido siempre relaciones complejas e intereses contradictorios. Si a ello se agregan otras motivaciones como el patriotismo, la obediencia a las jerarquías y la constitución psicológica de quienes desarrollan trabajos de investigación, el panorama ofrece un desafiante campo para la dilucidación de cuestiones más allá de la admonición pretenciosa de sedicentes filósofos o la pontificante obligación de adherir a códigos de conducta. Tanto la palabra de quienes nunca han desarrollado trabajo real de investigación con sujetos humanos como la imposición de dogmas o documentos reguladores son insuficientes para lograr que el producto de la investigación biomédica y psicosocial cumpla con los imperativos de ser técnicamente fiable y moralmente correcto. Este producto depende básicamente de la conciencia comunitaria.
Para aclarar este aserto es necesario aceptar que la ciencia y la técnica, aplicadas a los asuntos humanos, constituyen una práctica social en la que intervienen numerosos actores. El tópico del científico aislado que, obsesionado por encontrar la verdad, labora en la soledad de su laboratorio y dona sus descubrimientos para el bien de la humanidad hace ya tiempo dejó de ser popular.

La investigación moderna, tanto en su versión de big science, financiada por poderosos consorcios y realizada por numerosos científicos, como en el trabajo de laboratorio realizado por equipos pequeños, engendra una poderosa cultura que moldea desde la propaganda hasta la educación y se caracteriza por "subculturas epistémicas" de expertos que comparten métodos, procedimientos y lenguaje. Convencen corporativamente a otros agentes sociales de la importancia de sus aportaciones y logran apoyo para sus iniciativas a cambio de la promesa de rendimientos económicos, políticos o militares. Defienden sus fueros contra posturas alternativas y resguardan su capacidad de autorregulación celosamente.

Lo interesante del proceso de autonomización de los saberes técnicos es que, alcanzado cierto nivel de desarrollo social y del consiguiente poder, se desligan de las motivaciones originales y sirven a otros intereses.

Suele decirse que las sociedades cultivan la ciencia y las técnicas para obtener el poder del conocimiento, preparar 
a sus grupos dirigentes, competir el concierto mundial por prestigio y poder y asegurar progreso y desarrollo socioeconómico.

No siempre estas metas amplias son compartidas, o tan siquiera percibidas, por el grupo de quienes cultivan las ciencias. Tales argumentos sirven, es verdad, para lograr el reconocimiento y el apoyo sociales, pero sus motivaciones personales pueden estar distantes de estas altruistas metas.

En lo operativo, sin embargo, termina por imponerse un pensamiento estratégico, que ilustra el carácter de práctica social a que aludimos antes.

Esto es, la necesaria interrelación entre creatividad intelectual, apoyo político, financiamiento lucrativo y otros intereses.

Se produce el fenómeno que Lucien Sfez identifica como "supercodificación", por el cual se amalgaman intereses diversos que confluyen a decisiones trascendentes para ciertas áreas científicas (Sfez, 1995).

Es el caso del proyecto Manhattan y del Proyecto Genoma, que requirieron potentes inversiones y unificación de voluntades hasta convertirse en decisiones político-científicas de enorme magnitud, con consecuencias políticas, militares y económicas que sobrepasan el ámbito científico y obligan a repensar no solamente los medios sino también los fines de toda la institucionalidad científico-técnica.

\section{LA INVESTIGACIÓN CON Y EN SUJETOS HUMANOS}

En la bioética contemporánea, el debate sobre el consentimiento voluntario de los sujetos de la investigación tiene un destacado papel. Constituyó el primer artículo del llamado "código de Nürenberg", que sintetiza los resultados del juicio a los médicos y científicos alemanes por parte del tribunal estadounidense que juzgó sus actividades en el contexto del Reich nacionalsocialista. La "protección" de los individuos expuestos a la investigación o la manipulación terapéutica encontró expresión social en comisiones de estudio, formulación de principios y redacción de pautas de comportamiento. Los conceptos de "autonomía" y "consentimiento informado" pertenecen a los más citados y repetidos en la literatura internacional. Los avances jurídicos y legislativos de los países centrales obligan a la academia y la industria a reclutar sus "sujetos" en naciones más atrasadas educacional y tecnológicamente, con lo que se instaura un régimen de "doble estándar", permitiendo en algunos sitios prácticas que en otros están prohibidas. Por cierto, la protección de los sujetos no solamente implica su aceptación voluntaria de lo que se hará con ellos sino también la posibilidad de retirarse de los estudios y exigir compensaciones por daños y protección contra riesgos, además de beneficios al terminar las investigaciones (CIOMS, 2002). 
La frondosa literatura y los numerosos códigos y pautas relacionados con la investigación con sujetos humanos muestran que, salvo la reiteración de algunos puntos y la formulación más o menos ingeniosa de condiciones o restricciones (confidencialidad, beneficios, respeto a la dignidad) es conveniente aumentar el estudio empírico de lo que ocurre en el sistema social de la investigación con sujetos humanos, concebida como una forma institucionalizada de relación social (Bandres \& Tsuchida, 2004). En ella interesa su estructura, sus procesos, sus motivaciones, los intereses de sus participantes, la estabilidad de sus lazos, el papel de la emoción y los afectos, entre otros muchos temas.

A veces se reemplaza el término "sujeto" por el de "participante", en un esfuerzo cosmético por indicar que las personas en o sobre las que se realizan estudios no son simples medios (o no debieran serlo), mas ello no ha aumentado el interés por estudiar la díada investigador-sujeto como un sistema social. La "relación médico-paciente", en cambio, ha adquirido el rango de una verdadera institución social sobre la que ejercen su magisterio desde los científicos sociales hasta los médicos, pasando por una amplia gama de opinantes que pontifican sobre lo que debe ser y prescriben comportamientos, tanto para los profesionales (médicos, terapeutas) como para los pacientes (clientes, usuarios).
Por este motivo, procede examinar algunas condiciones a considerar en futuras investigaciones. Por simplicidad, nos concentraremos en las características, derechos y deberes de los sujetos (o participantes) de la investigación. La finalidad es que los investigadores desarrollen programas pertinentes en sus respectivos ámbitos y se establezca una colaboración de pesquisa.

\section{INVESTIGACIÓN TERAPÉUTICA Y NO TERAPÉUTICA}

Conviene aludir de entrada a una confusión frecuente. En la primera versión de la Declaración de Helsinki, promulgada por la Asociación Médica Mundial, se hablaba de dos tipos de investigación. Una -la llamada terapéuticaademás de producir beneficios sociales por aumento del conocimiento generalizable, era caracterizada por producir beneficios directos a los sujetos-pacientes. Así, un ensayo farmacológico podía ser, al mismo tiempo, una terapia adecuada y los miembros del grupo experimental podrían esperar curaciones o remisiones de sus dolencias. La otra investigación -no terapéutica- se caracterizaba por producir sólo conocimiento válido y generalizable que, posiblemente, beneficiaría a las personas en el futuro.

No es este el sitio para discutir en profundidad la lógica o las implicaciones de esta distinción que ha sido atacada desde diversos frentes. $\mathrm{Y}$ aunque 
es razonable como principio, puede llevar a lo que se conoce como "malentendido terapéutico", la confusión entre tratamiento e investigación que, en ocasiones, se usa soterradamente para reclutar sujetos de investigación. Forma parte así de los eventuales beneficios $\mathrm{u}$ ofrecimientos con que se convence a individuos para ser "voluntarios" o "participantes". La redacción de los documentos mediante los cuales se invita a las personas a participar en estudios e investigaciones puede en ocasiones inducir a confusión, deliberada o involuntariamente.

En el contexto de investigaciones biomédicas o psicosociales cabe reconocer que ellas pueden tener "momentos" terapéuticos y "momentos" puramente científicos. Difícil sería conducir un estudio sobre la eficacia de una forma de psicoterapia sin que la sola actividad no fuera ya, por sí misma, un factor modificador de las condiciones del estudio. Como enseguida veremos, los efectos simbólicos de intervenir no se reducen a los efectos placebo y Hawthorne e incluyen variables biográficas altamente individuales.

\section{EQUIDAD EN RIESGOS Y BENEFICIOS}

Relacionado con lo anterior está un fenómeno que se hizo muy notorio a raíz de la epidemia de sida. La mayoría de los documentos clásicos siempre aludió a la "protección" de los sujetos y la práctica convencional, bajo este princi- pio de precaución protectora, tendió a excluir ciertos grupos de la condición de sujetos experimentales. Así, hubo pocos datos sobre mujeres en edad fértil, niños pequeños y otros grupos, lo cual tiene también una dimensión negativa, pues lleva a una carencia de informaciones sobre ellos. Pero la existencia de una enfermedad en sus comienzos incurable, la desesperación por la estigmatización y la marginación, llevaron a muchos a pedir ser sujetos de experimentación en cualquier estudio que prometiera, aunque fuera remotamente, una posibilidad de alivio o curación. De allí nace un concepto peculiar, cual es el derecho a ser sujeto de experimentación, expresable como equidad en la composición de las muestras o en la repartición de riesgos $\mathrm{y}$ beneficios.

Casi no precisa indicarse que un sujeto que desea ardientemente participar en un estudio se encuentra en una disposición anímica muy distinta de aquel que solo lo hace por algún incentivo monetario o en relativa ignorancia de eventuales beneficios.

\section{Motivaciones Para SER SUJeto EXPERIMENTAL}

Frente al enorme despliegue de trabajos para caracterizar pacientes y al numeroso conjunto de estudios sobre las "buenas prácticas" que deben manifestar los investigadores, es escaso el análisis del "sujeto experimental". Descarte- 
mos aquellas personas que son inducidas a participar en estudios porque están en una relación de dependencia con el investigador o se acogen a la confianza que este les merece porque actúa como su terapeuta. De hecho, los estudios con prisioneros o estudiantes exigen una cautela especial y la demostración de que no se ha empleado coacción para que participen en un estudio. Ciertamente, lo que suele declararse en los protocolos es distinto de lo que ocurre en la realidad y merecería un análisis usando métodos indirectos. Aquí solo nos preguntamos por los voluntarios "sanos" o "normales" reclutados para estudios biomédicos o psicosociales.

La importancia de este análisis radica en la posibilidad de que la persona del sujeto, independientemente del diseño o de los fines de un estudio, influencie los resultados al punto de hacerlos no generalizables. Es un riesgo obvio en los estudios multicéntricos. En ellos, las condiciones particulares de un contexto pueden afectar los resultados, por ejemplo, mostrando mayores efectos placebo o mayor número de personas que abandonan los estudios. Otro factor aleatorio deriva de interacciones no deseadas entre investigador y sujeto, que pueden dar lugar a efectos o consecuencias difíciles de evaluar. Soterradamente, ciertos estereotipos sociales, prejuicios inconscientes o incluso rechazos no manifiestos hacia ciertas personas pueden influir en las relaciones interpersonales.
Obviamente, un voluntario tiene o puede tener un conjunto de intereses de índole personal. Van desde beneficios económicos hasta curiosidad, pasando por beneficios más sutiles como conocimiento de un ambiente distinto, afán de notoriedad o deseo de agradar. Frecuentemente se hace referencia a lo que constituye un estímulo genuino para diferenciarlo de lo que es soborno o directamente compra de servicios. Si una persona tiene un ingreso de cien dólares mensuales, es altamente probable que acepte participar en cualquier tipo de estudio que le ofrezca esa suma por un día de experimentos. La vulnerabilidad de las personas no conoce límites, toda vez que siempre es posible encontrarla, dependiendo de cómo se la busque. Las más obvias de la pobreza, la discapacidad o la enfermedad se mezclan con las debidas al histrionismo, afán de preeminencia o sensibilidad al halago.

La personalidad de los voluntarios ha sido siempre un tema controvertido. Por de pronto, quienes responden a un aviso de prensa solicitando interesados en participar en una investigación ya constituyen un subconjunto de todos los voluntarios posibles. Muchos son individuos que aman las sensaciones y probablemente puntuarían alto en las escalas de búsqueda de sensaciones (sensation seeking) o en las de extraversión, característica de individuos en quienes la acción precede a la reflexión y que actúan impulsivamente. 
La selección de sujetos para estudios plantea de suyo el problema de qué constituye un grupo. Es presumible que quienes comparten algunas características, como el sexo por ejemplo, puedan ser tratados de manera uniforme, pero ni siquiera ese atributo físico asegura uniformidad, toda vez que el género -como construcción social basada en el sexo biológico mas no reducible a él- puede ser muy diverso. Quienes se encuentran afectados de una enfermedad parecen naturalmente pertenecer a un grupo homogéneo, pero es evidente que el estadio en que se encuentre la dolencia, la red de apoyo social que cada individuo tiene, la concomitancia de otras afecciones o el tipo de seguro de salud introducen diversidad. Quienes habitan una misma región o pertenecen a una etnia definida pueden también ser considerados miembros de un grupo, pero - de nuevo- las diversidades intragrupo son inevitables.

Se impone la conclusión de que un grupo es siempre una construcción más o menos artificial y que la pureza de los métodos de muestreo siempre debe considerar una variabilidad que entra al factor error. Pocas veces, no obstante, se tiene en cuenta que un elemento importante es la tendencia a la participación voluntaria. De hecho, si se exami- nan los textos más usuales sobre ética de la investigación o sobre metodología, lo que se llama voluntariedad es la aceptación de condiciones impuestas por el diseño o por el investigador, no la expresión espontánea de un deseo. De allí que la voluntariedad aceptada en un formulario de consentimiento informado encubra desde entusiasmo y curiosidad de un sujeto en particular hasta desinterés, indiferencia y hasta repulsión de otro. Esta disposición interior queda rotulada de aceptación sin más con la firma del formulario o la disposición a participar.

Aparte, la importancia que esto puede tener en los resultados del estudio (por ejemplo, efectos placebo y Hawthorne $^{1}$ ), de la influencia sobre los resultados del tratamiento o la intervención (al llenar un cuestionario o informar efectos secundarios), en un plano macrosocial incide sobre la agregación de los datos. Por ejemplo, se puede tener una aproximación estudiando el número de personas que abandona el estudio, del mismo modo que se estima la eficacia de un tratamiento por los "drop-outs" que abandonan el estudio o aquellos que muestran escaso acatamiento (compliance) sin retirarse abiertamente.

Es sabido, por ejemplo, que en psiquiatría es muy distinto estudiar pobla-

1 En términos simples, este efecto es debido al hecho de sentirse observado/a o ser parte de un estudio. 
ciones de consultantes espontáneos que personas seleccionadas por encuestas en la población general. Si estas condiciones relacionadas con los sujetos no son incorporadas al análisis puede tenerse información errónea.

\section{LA AUTOEXPERIMENTACIÓN}

Un tipo especial de "voluntario" es aquel que investiga en sí mismo, ya sea por observaciones sistemáticas o sometiéndose a intervenciones propias o ajenas. La ficción literaria cuenta la historia del Dr. Jekyll y Mr. Hyde, aludiendo a las fuerzas que un investigador, deseoso de probar la certeza de sus estudios, puede liberar en el intento. Conocidos son los casos de médicos que se inocularon cepas de gérmenes virulentos o se sometieron a privación de alimentos o pidieron a colegas que les administraran substancias o los confinaran en ambientes especiales. Un análisis de quiénes son estas personas podría incluir desde el altruista deseo de enriquecer el acervo mundial hasta afán de sensaciones nuevas o notoriedad.

\section{CONSIDERACIONES FINALES}

Así como existe una cuidadosa taxonomía de pacientes y de los encuentros con sus terapeutas, todo un capítulo destinado a las características de la institución social de la díada investigadorsujeto está por escribirse en relación con la ética de la investigación que involucra sujetos humanos (Lolas et al.,
2002). Con el profesor Christian, durante mis estudios en Heidelberg, solíamos distinguir entre investigación "en" sujetos humanos e investigación "con" ellos (Lolas, 2001b). El segundo caso reconoce en el sujeto a un auténtico participante en una tarea común. El ser copartícipe, sin embargo, no siempre es positivo para la obtención de conocimiento válido o generalizable. Difícilmente se hubieran podido realizar las observaciones participantes de Goffman en hospitales psiquiátricos o los estudios de obediencia a la autoridad y tantos otros de saber los sujetos que eran sujetos o de haber consistido su aporte en ser voluntarios espontáneos. Hay que establecer un balance entre la necesidad de estudio objetivo y las percepciones, fantasías y deseos de los participantes.

De otra parte, la investigación biomédica y psicosocial ha estado señalada por el prejuicio objetivista, desdeñando métodos tales como la narrativa, que precisamente derivan conocimiento de las interacciones y de la construcción de significados en el contexto de experiencias. En este sentido, conviene hacer una distinción entre "experimento" y "experiencia" para separar el tipo de facticidad que es dable identificar en una investigación de orientación humanística (como muchas de psicología y psicoterapia). El experimento es una interrogación dirigida que manipula lo natural. La experiencia es la captación de lo natural en su plenitud y diversi- 
dad. Si el fin último de la investigación es predecir ocurrencias de fenómenos, ambas vías son posibles y, aún con las diferencias esperables de énfasis y estilo, debieran ambas generar certidumbres y predicciones.

Es necesario reconocer que muchas de las influencias no controlables sobre los resultados de la investigación debidas a caracteres o actitud de los sujetos han sido en ocasiones controladas a través del engaño (deception, en inglés). Esto es particularmente claro en algunos estudios psicológicos o sociales, en los que un conocimiento de los fines o contexto de la investigación hubiera alterado los resultados en formas no predecibles. La norma ética más usual es informar a los sujetos de modo tal que se minimicen estos riesgos. No obstante, debe reconocerse que este problema, aparte de plantear una dificultad metodológica y un conflicto ético, refuerza la necesidad de estudiar en mayor profundidad la persona del sujeto de experimentación y estudio en el contexto de la obtención de conocimiento generalizable y válido (López et al., 2003).

En este artículo solamente hemos querido llamar la atención sobre algunas consideraciones que destacan el valor de práctica social de la investigación que involucra sujetos humanos. Como tal práctica, no es nunca moralmente neutral y las motivaciones, emociones y rasgos psicológicos de sus actores y agentes merecen una sostenida atención.

\section{REFERENCIAS}

Bandres, P. \& Tsuchida, M. (2004). En investigación biomédica, un olvido histórico: La persona. Ars Medic, 9, 235242. Santiago de Chile.

Council of International Organizations for Medical Sciences (CIOMS) (2003). Pautas éticas internacionales para la investigación biomédica en sujetos humanos. Ginebra: CIOMS (Traducción de Unidad de Bioética OPS/OMS, Santiago de Chile).

Lolas, F. (2001a). Bioética. El diálogo moral en las ciencias de la vida. 2. ${ }^{a}$ edición. Santiago de Chile: Editorial Mediterráneo.

Lolas, F. (2001b). Paul Christian und die Heidelberger Schule. Fundamenta Psychiatrica (Schattauer Verlag), 15(4), 135-138.

Lolas, F., Valdebenito, C., \& Rueda, L. (2002). Decisiones éticas en investigación con seres humanos: aproximación al concepto de riesgo. Persona y Sociedad, 16(3), 321-326. Santiago de Chile.

López, M. J., Rueda, L., Valdebenito, C., Lolas, F. (2003). Ciudadanía y sujetos de investigación. Persona y Bioética, 7(18), 6-15. Bogotá.

Sfez, L. (1995). La santé parfaite-critique d'une nouvelle utopie. París: Editions du Seuil. 